\begin{tabular}{|c|l|}
\hline Title & Role of the dimerized gap due to anion ordering in spin-density wave phase of (TMT SF)2CIO4 at high magnetic fields \\
\hline Author(s) & Matsunaga, N.; Ayari and, A.; Monceau, P.; Ishikawa and, A.; Nomura, K.; W atanabe, M.; Y amada, J.; Nakatsuji, S. \\
\hline Citation & $\begin{array}{l}\text { PHY SICAL REVIEW B, 66(2), 024425 } \\
\text { https://doi.org/L0.1103/PhySRevB.66.024425 }\end{array}$ \\
\hline Issue Date & 2002-07-01 \\
\hline Doc URL & http://hdl.handle.net/2115/5741 \\
\hline Rights & Copyright $\odot 2002$ A merican Physical Society \\
\hline Type & article \\
\hline File Information & PRB66-2.pdf \\
\hline
\end{tabular}

Instructions for use 


\title{
Role of the dimerized gap due to anion ordering in spin-density wave phase of $(\mathrm{TMTSF})_{2} \mathrm{ClO}_{4}$ at high magnetic fields
}

\author{
N. Matsunaga* \\ Division of Physics, Hokkaido University, Sapporo 060-0810, Japan; \\ CRTBT-CNRS, Laboratoire Associé à l'UJF, BP 166, 38042 Grenoble, Cedex 9, France; \\ Grenoble High Field Laboratory, CNRS/MPI-FKF, BP 166, 38042 Grenoble, Cedex 9, France \\ A. Ayari and P. Monceau \\ CRTBT-CNRS, Laboratoire Associé à l'UJF, BP 166, 38042 Grenoble, Cedex 9, France \\ and Grenoble High Field Laboratory, CNRS/MPI-FKF, BP 166, 38042 Grenoble, Cedex 9, France \\ A. Ishikawa and K. Nomura \\ Division of Physics, Hokkaido University, Sapporo 060-0810, Japan \\ M. Watanabe, J. Yamada, and S. Nakatsuji \\ Department of Material Science, Himeji Institute of Technology, Kamigohri 678-1297, Japan
}

(Received 25 March 2002; published 18 July 2002)

\begin{abstract}
Magnetoresistance measurements have been carried out along the highly conducting $a$ axis in the fieldinduced spin-density wave (FISDW) phase of hydrogenated and deuterated (TMTSF) ${ }_{2} \mathrm{ClO}_{4}$ for various cooling rates through the anion ordering temperature. With increasing the cooling rate, (i) the high-field phase boundary $\beta_{\mathrm{HI}}$, observed at $27 \mathrm{~T}$ in hydrogenated samples for slowly cooled, is shifted towards a lower field, (ii) the last semimetallic SDW phase below $\beta_{\mathrm{HI}}$ is suppressed, and (iii) the FISDW insulating phase above $\beta_{\mathrm{HI}}$ is enhanced in both salts. The cooling rate dependence of the FISDW transition and of $\beta_{\mathrm{HI}}$ in both salts can be explained by taking into account the peculiar SDW nesting vector stabilized by the dimerized gap due to anion ordering.

DOI: 10.1103/PhysRevB.66.024425

PACS number(s): 75.30.Fv, 72.15.Gd, 74.70.Kn
\end{abstract}

\section{INTRODUCTION}

The quasi-one-dimensional (Q1D) organic compounds (TMTSF) ${ }_{2} X$, where TMTSF denotes tetramethyltetraselenafulvalene and $X=\mathrm{PF}_{6}, \mathrm{AsF}_{6}, \mathrm{ClO}_{4}$, etc., show many interesting phenomena such as superconductivity, anion ordering (AO), spin-density wave (SDW), a cascade of field-induced SDW (FISDW). ${ }^{1}$ The phase diagram of the FISDW phase in the $\mathrm{PF}_{6}$ salt, with quantized Hall resistance $\rho_{x y} \sim h /\left(n 2 e^{2}\right)$ in the sequence $n=\cdots 4,3,2,1,0$ as the magnetic field is increased, is successfully explained by the mean-field theory named the "standard model" based on the nesting of a pair of slightly warped parallel sheets of the Q1D Fermi surface. The states labeled with integer $n$ have been identified as semimetallic FISDW states while that with $n=0$ is a FISDW insulating state. However, the phase diagram of the FISDW phase in hydrogenated (TMTSF- $\left.h_{12}\right)_{2} \mathrm{ClO}_{4}$ (abbreviated as $h-\mathrm{ClO}_{4}$ hereafter) for the case of slow cooling is known to show disagreements with the standard model i.e., (i) in the low-field cascade of FISDW transitions, the sequence of Hall plateaus is not in the expected order. ${ }^{2}$ (ii) a very stable quantum Hall state is observed from 7.5 to $27 \mathrm{~T}^{3,4}$ (iii) the FISDW transition temperature $T_{\text {FISDW }}(\sim 5.5 \mathrm{~K})$ is independent of field above $15 \mathrm{~T}^{5}$ In order to explain the differences between $\mathrm{PF}_{6}$ and $\mathrm{ClO}_{4}$ salts, attention has been focused on the anion ordering which occurs in $h-\mathrm{ClO}_{4}$ at $24 \mathrm{~K}$ and dimerizes the system along the $b$ direction by a superlattice potential $V$ with a wave vector $Q=(0,1 / 2,0) .{ }^{6}$ This dimerization separates the original Fermi surface (FS) into two pairs of open orbit FS sheets. Although the FISDW phase diagram in the slowly cooled $h-\mathrm{ClO}_{4}$ salt is widely investigated experimentally and theoretically, however, it is still in question.

When the $h-\mathrm{ClO}_{4}$ salt is rapidly cooled through the anion ordering temperature $T_{\mathrm{AO}}$, the orientations of anions are frozen in two directions at random probability and the SDW phase is induced. In general, the deuteration of the TMTSF salt is thought to work as a positive chemical pressure in the crystal. ${ }^{7}$ When the positive chemical pressure by deuteration is applied to the $\mathrm{ClO}_{4}$ salt, the nesting of the Q1D Fermi surface becomes more imperfect and the stabilization of the SDW phase in the intermediate cooled states is suppressed. As a result, the deuterated (TMTSF- $\left.d_{12}\right)_{2} \mathrm{ClO}_{4}$ salt (abbreviated as $d-\mathrm{ClO}_{4}$ hereafter) is expected to show the FISDW phase in a broad range of cooling rates in contrast with the case of hydrogenated ones.

In this paper, we describe the cooling rate $\mathfrak{R}_{\mathrm{C}}$ dependence of the FISDW phase diagram for the hydrogenated, for the smallest cooling rate accessible: $0.0009 \mathrm{~K} / \mathrm{s}$, and deuterated, for different cooling rates: $0.0009,0.018$, and $0.67 \mathrm{~K} / \mathrm{s}, \mathrm{ClO}_{4}$ salts under strong magnetic fields applied parallel to the $c^{*}$ axis; we discuss the role of the dimerized gap due to AO controlled by the cooling rate, and that of the chemical pressure by deuteration in the FISDW phase diagram, and we compare the results in deuterated salts with those measured in hydrogenated ones. 


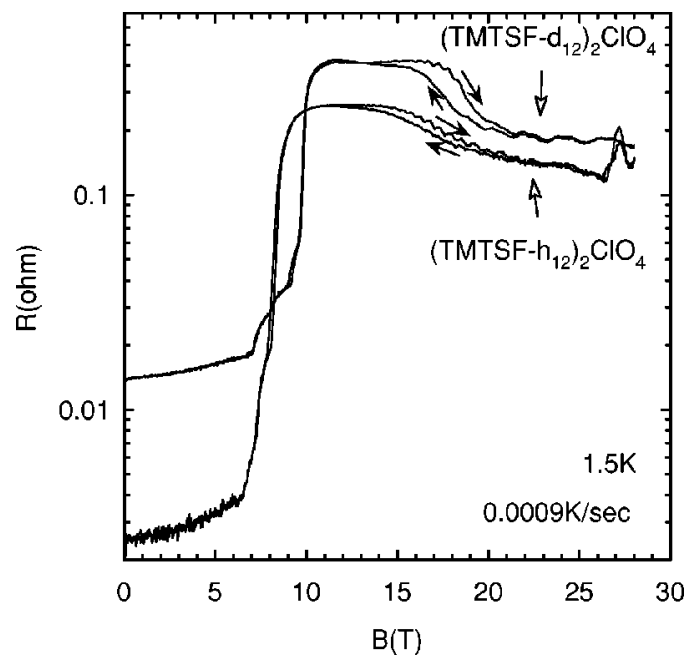

FIG. 1. Magnetoresistance along the highly conducting $a$ axis in hydrogenated and deuterated (TMTSF) ${ }_{2} \mathrm{ClO}_{4}$ for the relaxed state (cooling rate about $0.0009 \mathrm{~K} / \mathrm{s}$ ) with the magnetic field parallel to the lowest conductivity direction $c^{*}$ at $1.5 \mathrm{~K}$.

\section{EXPERIMENT}

Single crystals of (TMTSF) ${ }_{2} \mathrm{ClO}_{4}$ were synthesized by the standard electrochemical method. The resistance measurements along the conducting $a$ axis were carried out using a standard four-probe dc method over the temperature range from 1.5 to $10 \mathrm{~K}$. The typical size of the sample was 1 $\times 0.1 \times 0.1 \mathrm{~mm}^{3}$. Electric leads of $10 \mu \mathrm{m}$ gold wire were attached with silver paint onto gold evaporated contacts. The current contacts covered the whole areas of both ends of the sample for a uniform current. In order to prepare states with various degrees of anion ordering, the sample was heated up to $40 \mathrm{~K}$, and then cooled again with a progressive decrease of the heating to give a controlled cooling rate $\mathfrak{R}_{\mathrm{C}}$. The temperature was measured using a Cernox CX-1050-SD resistance thermometer calibrated by a capacitance sensor in magnetic fields. The measurements in the fields to $28 \mathrm{~T}$ were done in a resistive magnet at the Grenoble High Magnetic Field Laboratory.

\section{RESULTS AND DISCUSSION}

Figure 1 shows the magnetoresistance along the highly conducting $a$ axis in hydrogenated and deuterated (TMTSF) ${ }_{2} \mathrm{ClO}_{4}$ at $1.5 \mathrm{~K}$ for relaxed state, in which $\mathfrak{R}_{\mathrm{C}}$ is about $0.0009 \mathrm{~K} / \mathrm{s}$. Magnetic field $B$ up to $28 \mathrm{~T}$ was applied parallel to the lowest conductivity direction $c^{*}$. For hydrogenated $h-\mathrm{ClO}_{4}$, it is found from the sudden increase of resistance that the transitions to the first and last $(n=1)$ semimetallic SDW phase take place at about 6.5 and $8 \mathrm{~T}$, respectively. With increasing $B$, the nonoscillatory background resistance $R_{0}$ goes up and has a broad peak between 10 and $15 \mathrm{~T}$ and a decrease above $15 \mathrm{~T}$. The magnetoresistance shows hysteresis between 14 and $21 \mathrm{~T}$. The rapid oscillation (RO) is clearly seen above $14 \mathrm{~T}$ in Fig. 1. Above 27 $\mathrm{T}$ the field which was proposed by McKernan et al. as a new phase boundary $\beta_{\mathrm{HI}}$ of a first-order transition, ${ }^{5}$ both $R_{0}$ and the amplitude of RO suddenly increases; the magnetoresis-

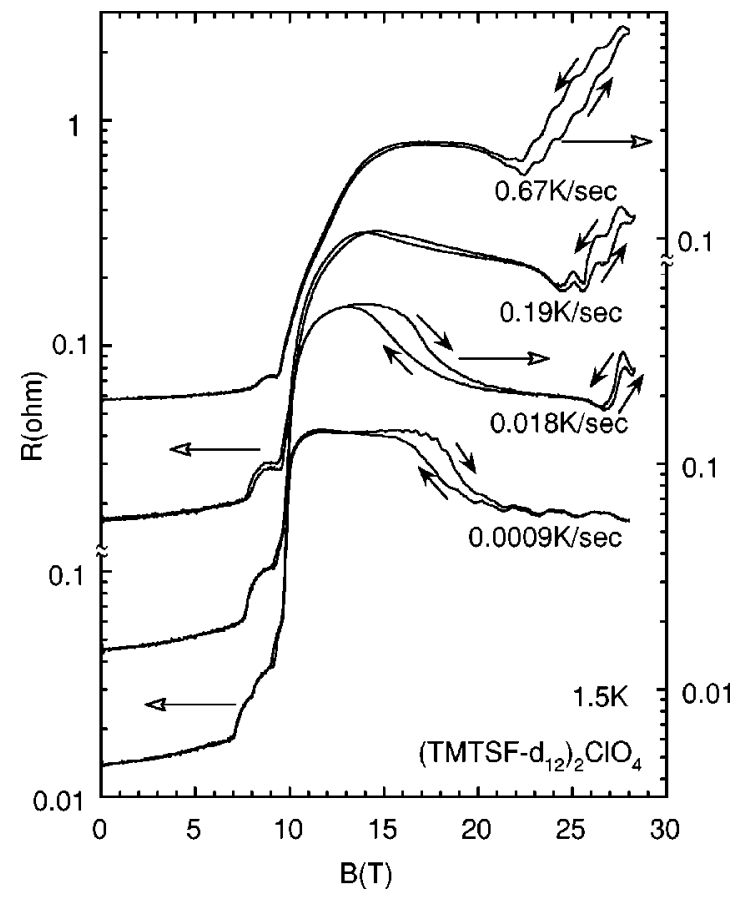

FIG. 2. Cooling rate dependence of the magnetoresistance of (TMTSF- $\left.d_{12}\right)_{2} \mathrm{ClO}_{4}$ with the magnetic field parallel to the $c^{*}$ direction at $1.5 \mathrm{~K}$.

tance shows an isosceles triangle shape. The sudden increase of $R_{0}$ and of the RO amplitude is consistent with previous results. ${ }^{8}$ On the other hand, by deuteration of the $\mathrm{ClO}_{4}$ salt, $T_{\mathrm{AO}}$ increases from 24 to $27 \mathrm{~K}$, and the transition field to the first and last semimetallic SDW phase increases from 6.5 to $7 \mathrm{~T}$ and from 8 to $9.7 \mathrm{~T}$, respectively. In addition, the field of the broad peak shifts to the high-field side and the phase boundary at high field $\beta_{\mathrm{HI}}$ is not observed below $28 \mathrm{~T}$. This indicates that deuteration of the $\mathrm{ClO}_{4}$ salt moves the FISDW phase boundary towards the high pressure side and $\beta_{\mathrm{HI}}$ is pushed out above $28 \mathrm{~T}$. These results are regarded as the consequence of a positive chemical pressure in the crystal by deuteration. ${ }^{9,10}$ This is consistent with usual deuteration effects. ${ }^{711}$ Moreover, the magnetoresistance of $d$ - $\mathrm{ClO}_{4}$ shows a steplike change from the phase between 10 and $17 \mathrm{~T}$ to the phase above $20 \mathrm{~T}$ with hysteresis between 14 and $21 \mathrm{~T}$. Although the possibility of a new phase boundary has been proposed around $17 \mathrm{~T}$ for $h-\mathrm{ClO}_{4}$ from magnetoresistance measurements, ${ }^{12}$ the origin of the above steplike magnetoresistance and hysteresis is unsolved.

The cooling rate $\mathfrak{R}_{\mathrm{C}}$ dependence of the magnetoresistance of $d-\mathrm{ClO}_{4}$ at $1.5 \mathrm{~K}$ is shown in Fig. 2. From this figure, we find that the transition field to the last semimetallic SDW at 9.7 $\mathrm{T}$ is not sensitive to $\mathfrak{R}_{\mathrm{C}}$. This means that $\mathfrak{R}_{\mathrm{C}}$ in this region dose not change the effective pressure in the crystal. With increasing $\mathfrak{R}_{\mathrm{C}}$, the sudden increase of the resistance at 9.7 $\mathrm{T}$ becomes rounded, and both the RO and the large hysteresis of the magnetoresistance becomes dim in the semimetallic SDW phase. Moreover, $\beta_{\mathrm{HI}}$ determined from the sudden increase of $R_{0}$ and of the RO amplitude is clearly visible and it is shifted towards a lower field with a large hysteresis in the magnetoresistance when $\mathfrak{R}_{\mathrm{C}}$ is increased. The increase 


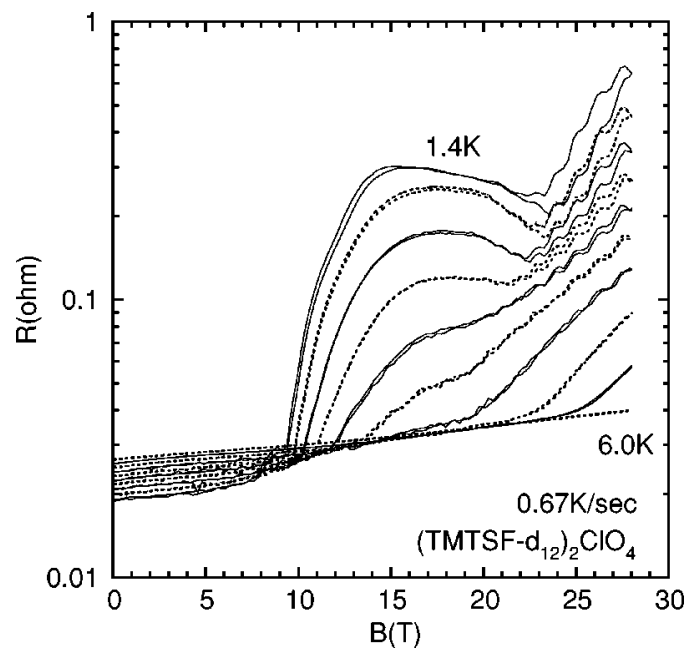

FIG. 3. Magnetoresistance of (TMTSF- $\left.d_{12}\right)_{2} \mathrm{ClO}_{4}$ with the field parallel to the $c^{*}$ direction for the cooling rate about $0.67 \mathrm{~K} / \mathrm{s}$ at $1.3,2.0,2.5,3.0,3.5,4.0,4.3,5.0,5.5$, and $6.0 \mathrm{~K}$.

of the hysteresis loop above $\beta_{\mathrm{HI}}$ with increasing $\mathfrak{R}_{\mathrm{C}}$ is a consequence of the broadening of the first-order transition by disorder effects. We have also measured the $\mathfrak{R}_{C}$ dependence of the magnetoresistance of $h-\mathrm{ClO}_{4}$ and observed that $\beta_{\mathrm{HI}}$ decreases from $27 \mathrm{~T}$ for $0.0009 \mathrm{~K} / \mathrm{s}$ to $21.5 \mathrm{~T}$ for $0.018 \mathrm{~K} / \mathrm{s}$, although $\beta_{\mathrm{HI}}$ becomes not very well defined for the cooling rate above $0.19 \mathrm{~K} / \mathrm{s}$. One can conclude that $\mathfrak{R}_{\mathrm{C}}$ dependence of $\beta_{\mathrm{HI}}$ is a common feature in $\mathrm{ClO}_{4}$ salts.

In Fig. 3, we show the magnetoresistance of $d-\mathrm{ClO}_{4}$ at various temperatures for $\Re_{C}$ about $0.67 \mathrm{~K} / \mathrm{s}$. At this cooling rate, for each temperature, we have determined the value of the FISDW transition of $d-\mathrm{ClO}_{4}$ as the intersection point between the extrapolations of the magnetoresistance curve in the FISDW and metallic phases. The FISDW transition temperature is plotted as a function of the magnetic field in Fig. 4. As shown in the upper-right side of Fig. 4, it increases with increasing the field $B$. This result is in agreement with previous measurements on $h-\mathrm{ClO}_{4}$ for $\mathfrak{R}_{\mathrm{C}}=0.5 \mathrm{~K} / \mathrm{s} .{ }^{13} \mathrm{We}$ have previously reported the quadratic increase of $T_{\text {FISDW }}$ with magnetic field above $12 \mathrm{~T}$ in $h-\mathrm{ClO}_{4}$ for $\mathfrak{R}_{\mathrm{C}}$ of 0.5 $\mathrm{K} / \mathrm{s} .{ }^{13}$ In the case of $d-\mathrm{ClO}_{4}, T_{\text {FISDW }}$ for $\Re_{\mathrm{C}}$ of $0.67 \mathrm{~K} / \mathrm{s}$ also roughly shows the quadratic field dependence above $13 \mathrm{~T}$. However, for intermediate cooling rates, the quadratic field dependence of $T_{\text {FISDW }}$ is not a quadratic increase as predicted by the mean-field theory ${ }^{14}$ but an envelope line of FISDW states with different quantum numbers $n$. We have also determined the value of the transition fields at $\beta_{\mathrm{HI}}$ from the intersection of the extrapolations of $R_{0}$ and have plotted them in the lower-right side of Fig. 4. As shown in Fig. 3, the large hysteresis in the magnetoresistance and $\mathrm{RO}$ are observed in the high-field phase region only below $3 \mathrm{~K}$. Above $3 \mathrm{~K}$, although the sudden increase of $R_{0}$ becomes rounded, the hump structure of $R_{0}$ exists still up to $4 \mathrm{~K}$. This means that $\beta_{\mathrm{HI}}$ exists up to $4 \mathrm{~K}$. The definition of our phase boundary is the same one determined by Naughton et al. ${ }^{4}$ as shown in Fig. 5. Below the magnetic field of this boundary, the nonzero Hall voltage was observed in all experiments. ${ }^{4,5}$

As a result, we show in Figs. 5 and 4 the FISDW phase

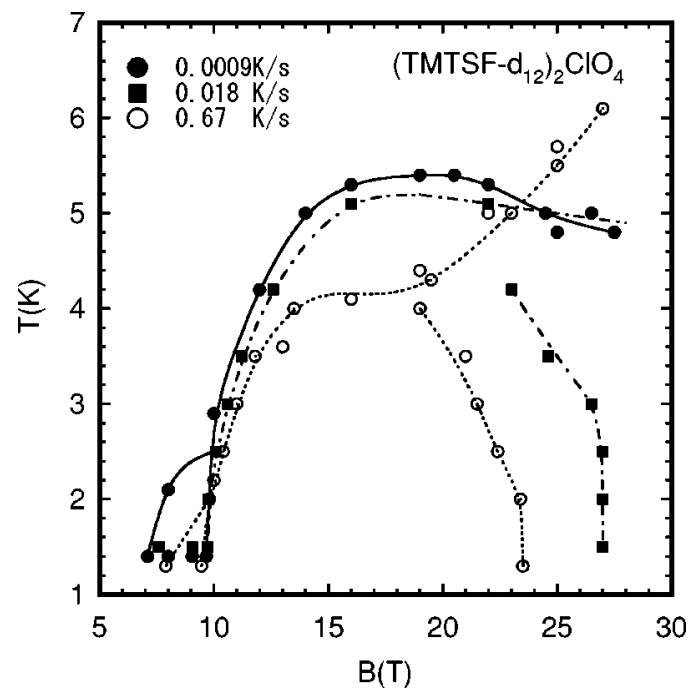

FIG. 4. The FISDW phase diagram in deuterated (TMTSF) ${ }_{2} \mathrm{ClO}_{4}$ constructed from many temperature and field sweeps for various cooling rates. The bold, dashed-dotted, and dashed lines are guides to the eye for $0.0009,0.018$, and $0.67 \mathrm{~K} / \mathrm{s}$ cooling rates, respectively.

diagram of $h-\mathrm{ClO}_{4}$ and $d-\mathrm{ClO}_{4}$ determined from magnetoresistance measurements for various $\mathfrak{R}_{\mathrm{C}}$, respectively. In Fig. 5 , solid lines show the phase boundary for the relaxed state proposed by McKernan et al. ${ }^{5}$ The dashed line is the phase boundary of the final FISDW phase for the relaxed state originally determined by Naughton $e t a l .{ }^{4}$ As shown in Fig. 5 , it is well known that $T_{\text {FISDW }}$ for a slow cooling in $h-\mathrm{ClO}_{4}$ is about $5.5 \mathrm{~K}$ and is almost independent of field $B$ above 15

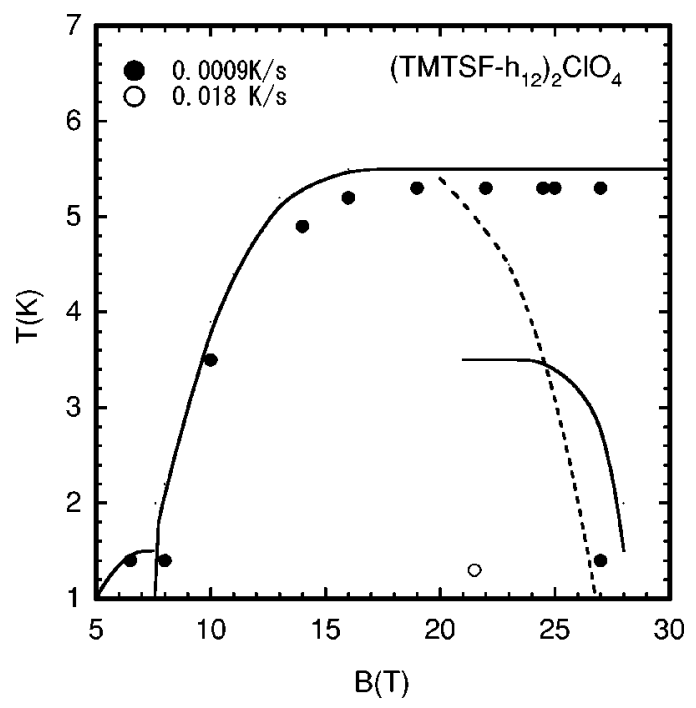

FIG. 5. The FISDW phase diagram in hydrogenated (TMTSF) ${ }_{2} \mathrm{ClO}_{4}$ with the magnetic field parallel to the lowest conductivity direction $c^{*}$. Solid lines show the phase boundary for the relaxed state proposed by McKernan et al. (Ref. 5). The dashed line is the phase boundary of the final FISDW phase for the relaxed state originally determined by Naughton et al. (Ref. 4). Solid and open circles indicate the FISDW transition temperature and the high-field phase boundary for our hydrogenated samples. 
T. We confirm this behavior for our hydrogenated samples as shown in Fig. 5. On the other hand, $T_{\mathrm{FISDW}}$ in $d-\mathrm{ClO}_{4}$ for $\mathfrak{R}_{\mathrm{C}}=0.0009 \mathrm{~K} / \mathrm{s}$ rapidly increases with increasing $B$ up to $16 \mathrm{~T}$ and slightly decreases above $20 \mathrm{~T}$ as shown in Fig. 4. The different behavior of $T_{\text {FISDW }}$ at high magnetic field between deuterated samples and hydrogenated ones is attributed to the difference of the chemical pressure in the crystal. It indicates that the negligible $B$ dependence of $T_{\text {FISDW }}$ above $15 \mathrm{~T}$ in hydrogenated samples is not intrinsic for slowly cooled (TMTSF) ${ }_{2} \mathrm{ClO}_{4}$. In $d-\mathrm{ClO}_{4}$ for $0.018 \mathrm{~K} / \mathrm{s}$, the $B$ dependence of $T_{\text {FISDW }}$ is almost the same as that for 0.0009 $\mathrm{K} / \mathrm{s}$. We observe, however, that $\beta_{\mathrm{HI}}(27 \mathrm{~T})$ which is independent of temperature below $2.5 \mathrm{~K}$ decreases with increasing temperature above $2.5 \mathrm{~K}$. Above $3.5 \mathrm{~K}, T_{\mathrm{FISDW}}$ has been determined from the hump structure of $R_{0}$ which exists up to $4.2 \mathrm{~K}$, although the sudden increase of $R_{0}$ becomes rounded. In $d-\mathrm{ClO}_{4}$ for $0.67 \mathrm{~K} / \mathrm{s}, \beta_{\mathrm{HI}}$ is observed at about $23.5 \mathrm{~T}$ and $T_{\text {FISDW }}$ increases with increasing $B$. The last semimetallic SDW phase between $9.7 \mathrm{~T}$ and $\beta_{\mathrm{HI}}$ is reduced from 5.5 to 4 $\mathrm{K}$ when the cooling rate $\mathfrak{R}_{\mathrm{C}}$ is increased. This result is consistent with the previous report ${ }^{13}$ for $h-\mathrm{ClO}_{4}$, the interpretation of which will be discussed later. Thus the experimental results lead to the conclusions that, with increasing the cooling rate $\mathfrak{R}_{\mathrm{C}}$, (i) the high field phase boundary $\beta_{\mathrm{HI}}$ shifts towards a lower field, (ii) the last semimetallic FISDW phase between $9.7 \mathrm{~T}$ and $\beta_{\mathrm{HI}}$ is suppressed, (iii) the FISDW insulating phase above $\beta_{\mathrm{HI}}$ in which the Hall voltage becomes almost zero ${ }^{5}$ is enhanced. These results mean that the last semimetallic FISDW phase and the FISDW insulating phase correspond to different FISDW states.

In order to explain this $\mathfrak{R}_{\mathrm{C}}$ dependence of the FISDW phase, we will now discuss the role of the dimerized gap due to anion ordering (AO) and that of the SDW nesting vector. As discussed in a previous report, ${ }^{13}$ the concentration of scattering centers associated with the boundaries between anionordered regions increases with increasing $\mathfrak{R}_{\mathrm{C}}$. This is consistent with the fact that the residual resistance in the metallic phase increases with increasing $\mathfrak{R}_{\mathrm{C}}$. For the slowly cooled $\mathrm{ClO}_{4}$ salt, AO creates a superlattice potential [Fig. 6(b) ] dividing the original Fermi surface into two pairs of open sheets. As a result, a dimerized gap due to AO is introduced in the electron band as shown in Fig. 6(a). Because the periodic anion potential is out of phase at the boundary between adjacent anion-ordered regions [Fig. 6(c)], for electrons moving across these boundaries, the dimerized gap due to AO is averaged out. These boundaries not only work as scattering centers but also suppress the dimerized gap due to AO. As a result, the effective dimerized gap due to AO decreases with increasing $\mathfrak{R}_{\mathrm{C}}$. Perturbative calculations using the standard model have shown that in the case of a small superlattice potential $V$ due to AO, the most stable SDW nesting vector $Q_{\mathrm{SDW}}$ is $\left(2 k_{F}, \pi / b\right)=\left(2 k_{F}, 0\right)$ [see Fig. 6(a) ] and subphases with odd quantum numbers, i.e., $\cdots 5,3,1$, successively appear when the field is increased. ${ }^{15,16}$ Although this model can explain the phase diagram of slowly cooled $h-\mathrm{ClO}_{4}$ below $8 \mathrm{~T}$ (Ref. 2) and the enhancement of the FISDW insulating phase above the high-field phase boundary $\beta_{\mathrm{HI}}$ with increasing $\mathfrak{R}_{\mathrm{C}}$, the decrease of $\beta_{\mathrm{HI}}$ and the suppression of the last semimetallic SDW phase with in-

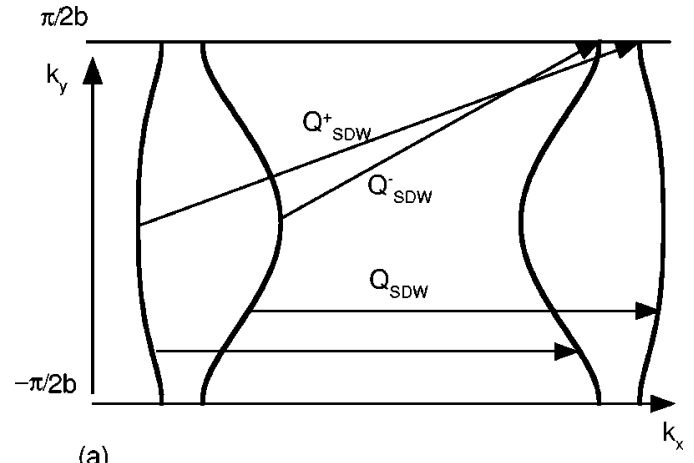

(a)



(b)

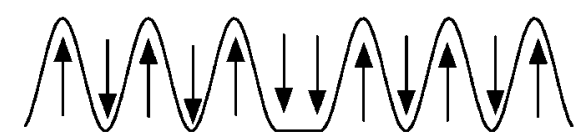

(c)

FIG. 6. (a) Schematic of the Fermi surfaces of (TMTSF) ${ }_{2} \mathrm{ClO}_{4}$, resulting from a dimerization of the system along the $b$ axis. $Q_{\mathrm{SDW}}=\left(2 k_{F}, \pi / b\right)=\left(2 k_{F}, 0\right)$ and $Q_{\mathrm{SDW}}^{ \pm}=\left(2 k_{F}^{ \pm}, \pi / 2 b\right)$ are the SDW nesting vectors. (b) Periodic anion potential without the boundary between anion ordered states. (c) Anion potential with the boundary between anion ordered states.

creasing $\Re_{\mathrm{C}}$ cannot be explained within the standard model with small $V$.

On the other hand, McKerman et al. proposed the nesting of another pair of the two pairs of open orbit Fermi surface sheets separated by the superlattice potential. ${ }^{5}$ Using nonperturbative calculations Kishigi claimed that a new FISDW phase with a SDW nesting vector $Q_{\mathrm{SDW}}^{ \pm}=\left(2 k_{F}^{ \pm}, \pi / 2 b\right)$ as shown in Fig. 6(a) is stabilized for a large $V \cdot{ }^{17}$ In fact, the magnitude of $V$ estimated from the angular dependent magnetoresistance measurements is of the order of the interchain hopping integral $\mathrm{t}_{b} \cdot{ }^{18}$ Recent calculations for $\mathrm{ClO}_{4}$ salt have pointed out that, when $V$ is increased, the SDW state with $Q_{\text {SDW }}$ is rapidly suppressed while the SDW state with $Q_{\text {SDW }}^{ \pm}$ becomes stable at higher value of $V \cdot{ }^{19,20}$ Because the FISDW phase between 12 and $24 \mathrm{~T}$ is suppressed with increasing $\mathfrak{R}_{\mathrm{C}}$, it is reasonable to estimate that the respective ground states of the last semimetallic FISDW phase and of the insulating FISDW phase are a $n=1$ state with $Q_{\mathrm{SDW}}^{ \pm}$and a $n$ $=0$ insulating state with $Q_{\text {SDW }}$. Because the FISDW phase with $Q_{\text {SDW }}^{ \pm}$becomes more stable with increasing the value of $V$, the model with $Q_{\mathrm{SDW}}^{ \pm}$can explain the decrease of $\beta_{\mathrm{HI}}$ and the suppression of the last semimetallic SDW phase. We are therefore led to conclude that $\beta_{\mathrm{HI}}$ of our experiment corresponds to the phase boundary between the $Q_{\mathrm{SDW}}^{ \pm}$phase and the $Q_{\text {SDW }}$ phase. As shown in Fig. 5, the high-field phase boundary $\beta_{\mathrm{HI}}$ (a first-order transition) proposed by McKernan et al. is located at $3.5 \mathrm{~K}$ between 21 and $25 \mathrm{~T}^{5}$ Their phase boundary is characterized by the step of Hall voltage and magnetization. They considered this phase boundary as 
the FISDW transition of one pair of Fermi surface within the $Q_{\text {SDW }}^{ \pm}$phase. As a result, the FISDW state at high magnetic fields can be separated three phases characterized by Hall voltage, magnetization, and the hump structure of $R_{0}$. This FISDW phase diagram is consistent with that calculated by Kishigi. ${ }^{17}$ Accordingly, if we assume that the last semimetallic FISDW phase is the FISDW phase with $Q_{\text {SDW }}^{ \pm}$stabilized by the dimerized gap due to $\mathrm{AO}$, the $\mathfrak{R}_{\mathrm{C}}$ dependence of the FISDW transition and of $\beta_{\mathrm{HI}}$ in the hydrogenated and deuterated $\mathrm{ClO}_{4}$ salts can be explained by the effective dimerized gap resulting from $\mathrm{AO}$.

\section{CONCLUSION}

We have measured the magnetoresistance, up to $28 \mathrm{~T}$, in the SDW phase of hydrogenated and deuterated (TMTSF) ${ }_{2} \mathrm{ClO}_{4}$, for various cooling rates $\mathfrak{R}_{\mathrm{C}}$ through the anion ordering temperature. We thus have obtained the $\mathfrak{R}_{\mathrm{C}}$ dependence of the FISDW transition temperature $T_{\text {FISDW }}$ and of the phase boundary at high-magnetic fields. The deuteration of the $\mathrm{ClO}_{4}$ salt works as a positive chemical pressure in the crystal and moves the phase boundaries of FISDW towards the high-field side. The deuterated (TMTSF- $\left.d_{12}\right)_{2} \mathrm{ClO}_{4}$ salt shows the FISDW phase in the wide cooling rate region which allowed us to investigate the $\mathfrak{R}_{\mathrm{C}}$ dependence of the FISDW phase in detail. We have found that, with increasing $\mathfrak{R}_{\mathrm{C}}$, the high-field phase boundary $\beta_{\mathrm{HI}}$ is shifted towards a lower magnetic field, the last semimetallic SDW phase below $\beta_{\mathrm{HI}}$ is suppressed, and the FISDW insulating phase above $\beta_{\mathrm{HI}}$ is enhanced. The $\mathfrak{R}_{\mathrm{C}}$ dependence of $T_{\mathrm{FISDW}}$ and of $\beta_{\mathrm{HI}}$ can be explained by the mean-field theory by taking into account the FISDW phase with the SDW nesting vector $Q \stackrel{ \pm}{\mathrm{SDW}}$ stabilized by the dimerized gap due to anion ordering.

\section{ACKNOWLEDGMENTS}

The authors wish to acknowledge helpful discussions with Dr. K. Kishigi. Some of this work was carried out as part of the "Research for the Future" project, JSPSRFTF97P00105, supported by the Japan Society for the Promotion of Science.
*Electronic address: mat@phys.sci.hokudai.ac.jp URL:http:// phys.sci.hokudai.ac.jp/LABS/nomura/english.html

${ }^{1}$ For a review, see T. Ishiguro, K. Yamaji, and G. Saito, Organic Superconductors II (Springer-Verlag, Berlin, 1998).

${ }^{2}$ M. Ribault, Mol. Cryst. Liq. Cryst. 119, 91 (1985).

${ }^{3}$ R. V. Chamberlin, M. J. Naughton, X. Yan, L. Y. Chiang, S.-Y. Hsu, and P. M. Chaikin, Phys. Rev. Lett. 60, 1189 (1988).

${ }^{4}$ M. J. Naughton, R. V. Chamberlin, X. Yan, S.-Y. Hsu, L. Y. Chiang, M. Ya. Azbel, and P. M. Chaikin, Phys. Rev. Lett. 61, 621 (1988).

${ }^{5}$ S. K. McKernan, S. T. Hannahs, U. M. Scheven, G. M. Danner, and P. M. Chaikin, Phys. Rev. Lett. 75, 1630 (1995).

${ }^{6}$ J. P. Pouget, G. Shirane, K. Bechgaard, and J. M. Fabre, Phys. Rev. B 27, 5203 (1983).

${ }^{7}$ K. Sinzger, S. Hunig, M. Jopp, D. Bauer, W. Bietsch, J. U. von Schutz, H. C. Wolf, R. K. Kremer, T. Metzenthin, R. Bau, S. I. Khan, A. Lindbaum, C. L. Lengauer, and E. Tillmanns, J. Am. Chem. Soc. 115, 7696 (1993).

${ }^{8}$ J. S. Brooks, R. G. Clark, R. H. McKenzie, R. Newbury, R. P. Starrett, A. V. Skougarevsky, M. Tokumoto, S. Takasaki, J. Yamada, H. Anzai, and S. Uji, Phys. Rev. B 53, 14406 (1996).
${ }^{9}$ F. Guo, K. Murata, H. Yoshino, S. Maki, S. Tanaka, J. Yamada, S. Nakatsuji, and H. Anzai, J. Phys. Soc. Jpn. 67, 3000 (1998).

${ }^{10}$ W. Kang, S. T. Hannahs, and P. M. Chaikin, Phys. Rev. Lett. 70, 3091 (1993).

${ }^{11}$ H. Schwenk, E. Hess, K. Andres, F. Wudl, and E. Aharon-Shalon, Phys. Lett. 102A, 57 (1984).

${ }^{12}$ Ok-Hee Chung, W. Kang, D. L. Kim, and C. H. Choi, Phys. Rev. B 61, 11649 (2000).

${ }^{13}$ N. Matsunaga, A. Briggs, A. Ishikawa, K. Nomura, T. Hanajiri, J. Yamada, S. Nakatsuji, and H. Anzai, Phys. Rev. B 62, 8611 (2000).

${ }^{14}$ G. Montambaux, Phys. Rev. B 38, 4788 (1988).

${ }^{15}$ A. G. Lebed and P. Bak, Phys. Rev. B 40, 11433 (1989).

${ }^{16}$ T. Osada, S. Kagoshima, and N. Miura, Phys. Rev. Lett. 69, 1117 (1992).

${ }^{17}$ K. Kishigi, J. Phys. Soc. Jpn. 67, 3825 (1998).

${ }^{18}$ H. Yoshino, A. Oda, T. Sasaki, T. Hanajiri, J. Yamada, S. Nakatsuji, H. Anzai, and K. Murata, J. Phys. Soc. Jpn. 68, 3142 (1999).

${ }^{19}$ D. Zanchi and A. Bjielis, Europhys. Lett. 56, 596 (2001).

${ }^{20}$ K. Sengupta and N. Dupuis, Phys. Rev. B 65, 035108 (2002). 